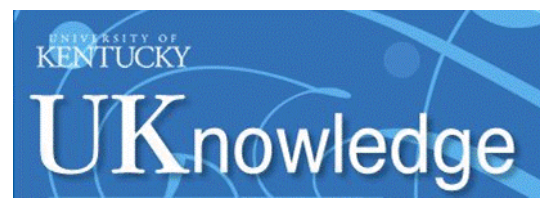

University of Kentucky

UKnowledge

$9-1-1998$

\title{
Polarization of Astronomical Maser Radiation. IV. Circular Polarization Profiles
}

Moshe Elitzur

University of Kentucky, moshe@pa.uky.edu

Follow this and additional works at: https://uknowledge.uky.edu/physastron_facpub

Part of the Astrophysics and Astronomy Commons, and the Physics Commons

Right click to open a feedback form in a new tab to let us know how this document benefits you.

\section{Repository Citation}

Elitzur, Moshe, "Polarization of Astronomical Maser Radiation. IV. Circular Polarization Profiles" (1998). Physics and Astronomy Faculty Publications. 220.

https://uknowledge.uky.edu/physastron_facpub/220

This Article is brought to you for free and open access by the Physics and Astronomy at UKnowledge. It has been accepted for inclusion in Physics and Astronomy Faculty Publications by an authorized administrator of UKnowledge. For more information, please contact UKnowledge@lsv.uky.edu. 


\section{Polarization of Astronomical Maser Radiation. IV. Circular Polarization Profiles}

Digital Object Identifier (DOI)

http://dx.doi.org/10.1086/306076

\section{Notes/Citation Information}

Published in The Astrophysical Journal, v. 504, no. 1, p. 390-395.

(c) 1998. The American Astronomical Society. All rights reserved.

The copyright holder has granted permission for posting the article here. 
The Astrophysical JouRnal, 504:390-395, 1998 September 1

(C) 1998. The American Astronomical Society. All rights reserved. Printed in U.S.A.

\title{
POLARIZATION OF ASTRONOMICAL MASER RADIATION. IV. CIRCULAR POLARIZATION PROFILES
}

\author{
MOSHE EliTZUR \\ Department of Physics and Astronomy, University of Kentucky, Lexington, KY 40506-0055; moshe@pa.uky.edu \\ Received 1997 December 24; accepted 1998 April 3
}

\begin{abstract}
Profile comparison of the Stokes parameters $V$ and $I$ is a powerful tool for maser data analysis, which provides the first direct methods for unambiguous determination of (1) the maser saturation stage, (2) the amplification optical depth and intrinsic Doppler width of unsaturated masers, and (3) the comparative magnitudes of Zeeman splitting and Doppler line width. Circular polarization recently detected in $\mathrm{OH}$ $1720 \mathrm{MHz}$ emission from the Galactic center appears to provide the first direct evidence for maser saturation.

Subject headings: line: profiles - magnetic fields - masers - polarization — radiative transfer
\end{abstract}

\section{INTRODUCTION}

Few properties of astronomical masers are determined directly by observations, most are inferred indirectly. Foremost among the latter is the maser saturation stage. Saturation has a significant impact on maser growth, so determining whether a maser is saturated $\left(J>J_{s}\right.$, where $J$ is the angle-averaged intensity and $J_{s}$ is the saturation intensity) or not is usually a precondition for analysis of the observations. Unfortunately, this crucial issue is not convincingly settled. Strong masers are generally believed to be saturated, but in most cases the evidence is less than compelling since it relies primarily on plausibility arguments rather than quantitative tests (see, e.g., Elitzur 1992, § 8.6). This unsatisfactory situation reflects a fundamental difficulty - neither $J$ nor $J_{s}$ is directly measurable. The saturation parameter $J_{s}$ is a theoretical quantity, determined only within the context of a given pumping scheme. And because maser radiation is highly beamed, $J=I \Omega / 4 \pi$ so this quantity, too, cannot be directly measured; the intensity $I$ is measurable when the maser is resolved, but the beaming angle $\Omega$ is unobservable. Similarly, the amplification optical depth has never been directly determined for any maser that amplifies its own radiation.

Recent VLA observations of $\mathrm{OH} 1720 \mathrm{MHz}$ masers near the Galactic center by Yusef-Zadeh et al. (1996) open up new possibilities for direct determination of some maser properties. Significant circular polarization (upward of $20 \%$ ) is detected in various spectral features, and the rightand left-hand components coincide on the sky, as expected from the Zeeman effect. Furthermore, the spectral shape of the Stokes parameter $V$ follows an antisymmetric $\mathrm{S}$ curve with sharp reversal at line center, the typical profile for Zeeman shift $\Delta v_{B}$ much smaller than the Doppler line width $\Delta v_{D}$. Similar results were previously reported for $\mathrm{H}_{2} \mathrm{O}$ masers in star-forming regions by Fiebig \& Gusten (1989) and for OH $1612 \mathrm{MHz}$ masers in OH/IR stars by Zell \& Fix (1991), but the polarization was lower and the quality of the data not nearly as high.

The general maser polarization solution was recently derived for arbitrary values of

$$
x_{B}=\frac{\Delta v_{B}}{\Delta v_{\mathrm{D}}}
$$

(Elitzur 1996, hereafter E96), and the solution properties at $x_{B} \ll 1$ closely match the observed circular polarization.
Here I show that a comparative analysis of the spectral profiles of $I$ and $V$, two measurable independent maser intensities, offers direct determination of various maser properties, in particular the saturation stage. The analysis is readily performed with the aid of the ratio profile

$$
\mathscr{R}(v)=\frac{V(v)}{I^{\prime}(v)}=\frac{v(v)}{I^{\prime}(v) / I(v)},
$$

where the prime denotes derivative with respect to frequency and $v=V / I$ is the fractional circular polarization. When $x_{B} \ll 1$, spectral analysis of $\mathscr{R}$ offers intrinsic sensitivity of order $x_{B}$ and has long been an important tool in studies of the Zeeman effect of thermal radiation (see, e.g., Troland $\&$ Heiles 1982). In that case $\mathscr{R}$ is constant across the spectral line and its magnitude determines the magnetic field along the line of sight. This constancy of $\mathscr{R}$ follows from some simple, general symmetry arguments as shown by Crutcher et al. (1993) (see also $\S 3$ below). However, maser exponential amplification during unsaturated growth destroys both the underlying symmetry and the constancy of $\mathscr{R}$, the saturation process restores both. The key to the different behavior, and $\mathscr{R}$ profiles, in the two regimes is the narrowing of the maser line during unsaturated amplification and its rebroadening during saturation.

The important differences between thermal and maser polarization are discussed in detail below. For completeness, some basic elements of the polarization theory developed in E96 are reproduced in $\S 2$. The $\mathscr{R}$ profile is discussed in $\S 3$ for thermal radiation and in $\S 4$ for maser radiation when $x_{B} \ll 1$. In $\S 5$, circular polarization for fully resolved Zeeman patterns, $x_{B}>1$, is discussed. The implications for observations are discussed in detail in $\S 6$.

\section{GENERALITIES}

A full description of electromagnetic radiation involves the 4-vector of its Stokes parameters $S=(I, Q, U, V)$. The general transfer equation for $S$ is

$$
\frac{d \boldsymbol{S}}{d l}=\epsilon+\boldsymbol{K} \cdot \boldsymbol{S},
$$

where $\epsilon$ is the 4-vector analog of the familiar volume emission coefficient and $\boldsymbol{K}$ is the matrix analog of the absorption coefficient. In the case of line radiation, $\boldsymbol{K}$ was derived in Litvak (1975) and is reproduced here by means of the notation of E96. For any spin transition, the radiative inter- 
actions with photons of polarization $\Delta m(=+1,0,-1)$ are characterized by an absorption coefficient $\kappa^{\Delta m}$. If we introduce

$$
\kappa^{1}=\frac{1}{2}\left(\kappa^{+}+\kappa^{-}\right),
$$

the mean absorption coefficient for $\Delta m= \pm 1$ transitions, and the three linear combinations

$$
\begin{aligned}
\kappa_{m} & =\frac{1}{2}\left[\kappa^{1}\left(1+\cos ^{2} \theta\right)+\kappa^{0} \sin ^{2} \theta\right] \\
\kappa_{l} & =\frac{1}{2}\left(\kappa^{1}-\kappa^{0}\right) \sin ^{2} \theta \\
\kappa_{c} & =\frac{1}{2}\left(\kappa^{+}-\kappa^{-}\right) \cos \theta,
\end{aligned}
$$

where $\theta$ is the angle of the radiation propagation direction from the quantization axis, taken here along the magnetic field, then the transfer matrix for line radiation is

$$
\boldsymbol{K}=\left(\begin{array}{cccc}
\kappa_{m} & \kappa_{l} & 0 & \kappa_{c} \\
\kappa_{l} & \kappa_{m} & 0 & 0 \\
0 & 0 & \kappa_{m} & 0 \\
\kappa_{c} & 0 & 0 & \kappa_{m}
\end{array}\right)
$$

The polarization of the 4-vector $S$ is characterized by $\Pi=(q, u, v)$, the 3-vector of its normalized Stokes parameters $q=Q / I, u=U / I, v=V / I$. It is easy to verify that the transfer matrix $K$ preserves full polarization, $|\Pi|=1$. From Maxwell's equations, radiation is always emitted fully polarized and for any intensity $I$ there is an ensemble of configurations of $\boldsymbol{S}$ (polarization modes) that differ from each other only by the directions of their corresponding unit vectors $\Pi$, i.e., the sense of polarization. For each mode, $\Pi$ remains a unit vector and radiative transfer only rotates it, transferring polarization between the linear and circular polarizations of individual modes. The polarization vector of the entire radiation field at intensity $I$ is obtained from the ensemble average of all polarization modes. This average differs from zero, and the observed radiation is polarized, only when certain modes are favored.

The emission term for the mode with initial polarization $\Pi_{0}$ can be written as

$$
\epsilon=\kappa_{m} S_{0}, \quad \text { where } S_{0}=S_{0}\left(1, \Pi_{0}\right),
$$

and $S_{0}$ is the standard source function. The ensemble average of these source terms has the common intensity $S_{0}$, and when the individual polarizations point at random directions in $q, u, v$ space it also has zero polarization. This is usually the case for radiation generated in spontaneous decays. The radiative transfer equations admit the formal solution for the intensity of individual modes

$$
I=S_{0} e^{\tau_{m}+\tau_{\pi}}
$$

where

$$
\tau_{m}=\int \kappa_{m} d l, \quad \tau_{\pi}=\int\left(\kappa_{l} q+\kappa_{c} v\right) d l .
$$

The optical depth $\tau_{\pi}$ describes the rotation of the mode polarization, and the growth of its intensity is dominated by $\tau_{m}$, which is always larger than $\tau_{\pi}$. Modes that have $\tau_{\pi}=0$ do not rotate, this provides a stationary polarization configuration.

\section{THERMAL CIRCULAR POLARIZATION}

In the case of thermal emission, the emerging radiation samples no more than $\sim 1$ optical depth into the source and its properties primarily reflect those of the source terms.
Therefore, only the polarization of the emission terms needs be considered; the subsequent effect of radiative transfer is generally only a perturbation.

In the presence of a magnetic field, thermal emission is circularly polarized and the polarization can be derived from rather general considerations (e.g., Crutcher et al. 1993). The energies of magnetic sublevels are shifted by the Zeeman effect, splitting each line into components with $\Delta m=-1,0,+1$ (where $\Delta m$ refers to the $m$ change in absorption for compatibility with the previous section). The standard Zeeman emission pattern is comprised of three components centered on the frequencies $v_{0}-\Delta m \Delta v_{B}$, each one with appropriate polarization properties. From symmetry, for $m$-independent line excitations the intensities of the three components are related via

$$
I^{ \pm}(x)=I^{0}\left(x \pm x_{B}\right) \simeq I^{0}(x) \pm x_{B} \frac{d I^{0}(x)}{d x},
$$

where $x=\left(v-v_{0}\right) / \Delta v_{\mathrm{D}}$ is the dimensionless frequency shift from line center. The last approximation is valid for all $x$ when $x_{B} \ll 1$. Because of the incoherence of thermal emission, the Stokes parameters are simple superpositions of the component intensities

$$
\begin{aligned}
& I(x)=\frac{1}{4}\left[\left(I^{+}+I^{-}\right)\left(1+\cos ^{2} \theta\right)+2 I^{0} \sin ^{2} \theta\right] \simeq I^{0}(x) \\
& V(x)=\frac{1}{2}\left(I^{+}-I^{-}\right) \cos \theta \simeq x_{B} \frac{d I^{0}(x)}{d x} \cos \theta,
\end{aligned}
$$

where the final expressions are the terms to leading order in $x_{B}$. Therefore,

$$
V(v)=\Delta v_{B} \cos \theta I^{\prime}(v), \text { i.e., } \mathscr{R}(v)=\Delta v_{B} \cos \theta
$$

(note that $x_{B} d / d x=\Delta v_{B} d / d v$ ). The constancy of the ratio profile $\mathscr{R}$ reflects the symmetry of equation (10) - the three $I^{\Delta m}$ are described by a single spectral profile at appropriately shifted arguments. This symmetry is unique to thermal radiation. The general symmetry of the problem is invariance under mirror reflections perpendicular to the magnetic axis and its consequence is instead

$$
I^{+}\left(v_{0}+\delta v\right)=I^{-}\left(v_{0}-\delta v\right)
$$

for any $\delta v$. Equation (10) follows only if the spectral shape of each $I^{\Delta m}$ is additionally symmetric about its centroid at $v_{0}-\Delta m \Delta v_{B}$. This is the case for thermal radiation because the emission is invariant under reversal of the particle motions.

\section{MASER CIRCULAR POLARIZATION}

In contrast with thermal emission, the Stokes parameters of maser radiation are dominated by the interaction terms in equation (3) rather than the source terms when $J \gtrsim$ $\left(S_{0} J_{s}\right)^{1 / 2}$ (E96). This condition is obeyed by virtually all bright maser sources. The polarization properties of radiation generated in spontaneous decays and in stimulated emissions are entirely different from each other. Consider for example a photon produced in a $\Delta m=0$ decay and thus linearly polarized. This photon contributes only to the linear polarization of the source terms and will be reflected as such in the polarization properties of observed thermal radiation. However, this photon can induce transitions with any value of $\Delta m$, thus any polarization, because it can also be described as a coherent mixture of two circularly pol- 
arized photons. Consider, for instance, the stimulated emissions induced by this photon in the case of a spin $1 \rightarrow 0$ transition. When the interacting particle is in the $m=0$ state, the induced photon is linearly polarized because it, too, is generated in a $\Delta m=0$ transition. But when the particle is in one of the $|m|=1$ states, the induced photon is circularly polarized because it is generated in a $\Delta m= \pm 1$ transition (the interaction amplitude is reduced, though). A photon generated in stimulated emission has the same wavevector as the parent photon but not necessarily the same polarization because the latter depends also on the magnetic quantum number of the interacting particle. Because of the finite line widths, different $\Delta m$ transitions overlap when $x_{B} \ll 1$ and particles in the same magnetic sublevel can interact with photons of different polarizations. Amplification by stimulated emission mixes the original polarizations of all the photons created in spontaneous decays.

The polarization mixing effect of the amplification process is reflected in the linear combinations in equations (5) and (6) and is the reason for the rotation of mode polarization. After amplification, any polarization the original radiation might have had is irrelevant. For example, even for thermal emission in a magnetic field polarized according to equation (12), each mode contains random linear polarization (arbitrary $q$ and $u$ ) for propagation in any direction other than $\theta=0$. Because the projection of every $\Pi$ on the $q-u$ plane points at a random direction, each mode rotates differently during the amplification process, individual polarizations are randomized, and the initial overall polarization disappears once the radiation field is dominated by the induced photons. The only polarization that can survive the amplification process is that of stationary modes that do not rotate, locking the individual polarization vectors. All four Stokes parameters then grow at the same rate and the fractional polarization remains constant. The stationary modes of amplified radiation are the eigenvectors of the matrix $K$ and were identified in E96. We denote by $\kappa_{0}(x)$ the unsaturated absorption coefficient of the maser transition in the absence of magnetic fields. In the presence of a magnetic field, the unsaturated absorption coefficient of the $\Delta m$ transition becomes

$$
\kappa_{0}^{\Delta m}(x)=\kappa_{0}\left(x+x_{B} \Delta m\right) \simeq \kappa_{0}(x)+x_{B} \Delta m \frac{d \kappa_{0}(x)}{d x},
$$

similar to equation (10) for the corresponding intensities. Again, the last relation is appropriate for $x_{B} \ll 1$. Maser polarization properties are controlled by the two ratios

$$
\begin{aligned}
& R_{1}=\frac{\kappa_{0}^{+}+\kappa_{0}^{-}}{2 \kappa_{0}^{0}} \simeq 1 \\
& R_{c}=\frac{\kappa_{0}^{+}-\kappa_{0}^{-}}{2 \kappa_{0}^{0}} \simeq \frac{x_{B}}{\kappa_{0}(x)} \frac{d \kappa_{0}(x)}{d x} .
\end{aligned}
$$

In each case, the last expression is the result to leading order in $x_{B}$, with the corrections smaller by order $x_{B}^{2}$ because of symmetry. The solution for the maser circular polarization when $x_{B} \ll 1$ is

$$
v_{0}(v)=\frac{8 R_{c}}{\left(2 R_{1}+1\right) \cos \theta} \simeq \frac{8 \Delta v_{B}}{3 \cos \theta} \frac{\kappa_{0}^{\prime}(v)}{\kappa_{0}(v)},
$$

where $\theta$ is restricted by $(2 / 3)^{1 / 2} \geq \cos \theta \geq 4(2)^{1 / 2} x_{B} x$ (see E96, eqs. [4.13]-[4.15]). The last expression is obtained by inserting $R_{1}$ and $R_{c}$ from the previous equation, corrections are smaller by order $x_{B}^{2}$.

\subsection{Unsaturated Masers}

When the maser is unsaturated $\left[J(v) \ll J_{s}\right]$, to leading order in both $J / J_{s}$ and $x_{B}, \kappa_{m} \simeq \kappa_{0}$ and $\kappa_{l}=\kappa_{c}=0$ for any mode, as is evident from equations (5) and (14). In this approximation the polarization rotation is neglected and the intensity follows the familiar solution of an unsaturated scalar maser $I(v)=S_{0} \exp \tau(v)$, where $\tau(v)=\kappa_{0}(v) \ell$ is the amplification optical depth, irrespective of polarization. Therefore $I^{\prime} / I=\tau \kappa_{0}^{\prime} / \kappa_{0}$, and for any polarization $v$

$$
\mathscr{R}(v)=\frac{v(v)}{\tau(v)} \frac{\kappa_{0}(v)}{\kappa_{0}^{\prime}(v)} .
$$

If we insert the stationary circular polarization from equation (16), the $\mathscr{R}$ profile of the maser solution in the unsaturated regime is

$$
\mathscr{R}(v)=\frac{8 \Delta v_{B}}{3 \cos \theta} \Psi(v),
$$

where

$$
\Psi(v)=\frac{1}{\tau(v)}=\frac{1}{\tau_{0}+\ln \left[I(v) / I\left(v_{0}\right)\right]} .
$$

The profile $\Psi(v)$ expresses the spectral shape of (the unmeasurable) $\tau(v)$ in terms of the measured intensity $I(v)$ and the free parameter $\tau_{0}=\tau\left(v_{0}\right)$, the maser optical depth at line center. This profile increases toward the line wings from a minimum at line center, fundamentally different from the flat profile of thermal radiation. When such $\mathscr{R}$ spectral shape is detected the maser is unsaturated; therefore the spectral profile of $\ln I(v)$ can be used to deduce the profile of $\kappa_{0}(v)$. The $\kappa_{0}$ profile determines the intrinsic Doppler line width, a quantity that is not known a priori and that is yet to be reliably determined in any maser source. Furthermore, the spectral relation between $\mathscr{R}(v)$ and $\ln I(v)$ provides a method for direct determination, the first of its kind, of the maser optical depth $\tau_{0}$ from observable quantities. Once $\tau_{0}$ is found, $\mathscr{R}\left(v_{0}\right)$ can be used to determine $B / \cos \theta$.

It may be noted that the polarization vector of the maser solution is not fully stationary during the early stages of maser growth when $J / J_{s}<x_{B}^{2}$; indeed, no polarization mode can avoid rotation at that stage. An estimate of this early rotation can be obtained by including the terms linear in $x_{B}$ while maintaining zeroth order in $J / J_{s}$. In this approximation order $\kappa_{m}$ remains equal to $\kappa_{0}$ so the intensity growth is the same, and $\kappa_{l}$ still is zero so the linear polarization does not rotate. However, $\kappa_{c} \neq 0$ and the circular polarization does vary according to

$$
\frac{d v}{d \tau}=\left(1-v^{2}\right) R_{c} \cos \theta .
$$

The solution of this equation for any initial polarization $v_{i}$ is

$$
v=\frac{v_{i}+\tanh \tau R_{c} \cos \theta}{1+v_{i} \tanh \tau R_{c} \cos \theta},
$$

a solution valid as long as $\exp \left(\tau_{0}\right) S_{0} / J_{s}<x_{B}^{2}$. Pumping schemes of $\mathrm{OH}$ masers typically have $S_{0} / J_{s} \sim 10^{-5}$ and the observed $20 \%$ polarization is reproduced with $x_{B}=0.03$ for propagation at $\cos ^{2} \theta=\frac{1}{3}$. Therefore this solution holds for $\tau_{0} \lesssim \ln (100)=4$.6. Figure 1 displays the $\mathscr{R}$ profiles of the 


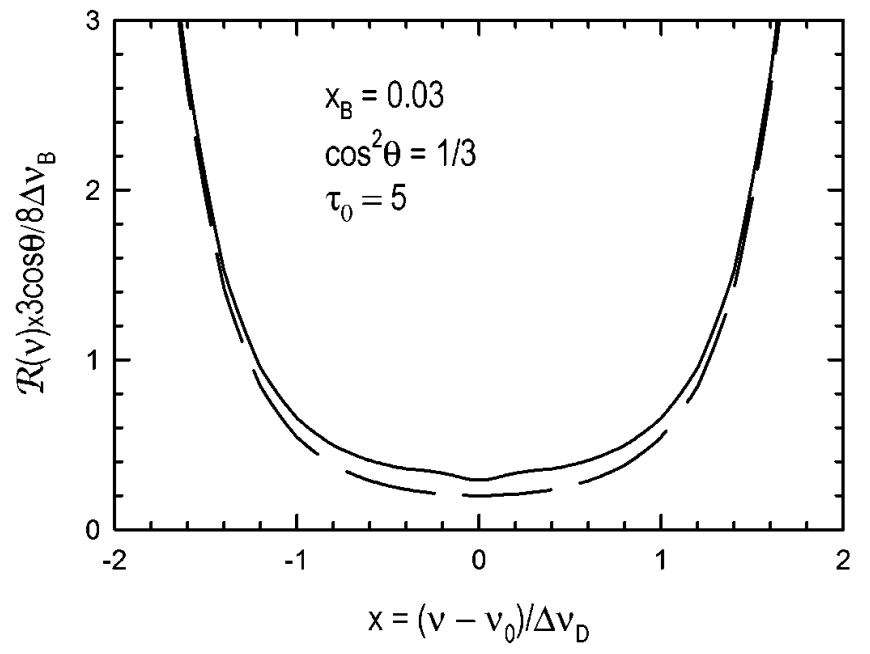

FIG. 1.- $\mathscr{R}$ profile of the polarization solution for an unsaturated maser with the parameters listed. The full line is the profile including the maximum possible polarization rotation (eq. [21]), the dashed line is the profile neglecting this rotation (eq. [19]). When the maser saturates, $\mathscr{R}$ becomes flat.

polarization solution $\left(v_{i}=v_{0}\right)$ at $\tau_{0}=5$ with and without the rotation. The difference between the two is practically insignificant. Furthermore, note that the neglected terms of higher order in $J / J_{s}$ will push the polarization toward stationary behavior and thus decrease the variation of $v$ below the result of equation (20). Therefore, this approximation produces an upper limit on variation of $v$ and the actual solution must fall between the two displayed profiles. As $\tau_{0}$ increases, the linear polarization joins the rotation so that the overall rotation of the polarization solution decreases further in inverse proportion to $J$ and the stationary approximation becomes even better.

The deviation from flatness of the maser $\mathscr{R}$ profile is a general result, reflecting line narrowing during unsaturated growth (e.g., Elitzur $1992 \S 4.5)$. It is instructive to examine the unsaturated amplification of polarized thermal radiation with an arbitrary intensity profile $I_{\mathrm{th}}(v)$ and a constant $\mathscr{R}$ profile, i.e., $V \propto I^{\prime}$. Equal amplification of the Stokes parameters produces

$$
I(v)=I_{\mathrm{th}}(v) e^{\tau(v)}, \quad V(v)=\Delta v_{B} \cos \theta I_{\mathrm{th}}^{\prime}(v) e^{\tau(v)} .
$$

Evidently, $V$ cannot remain proportional to $I^{\prime}$ because the derivative of the amplified intensity is not equal to the amplified intensity derivative. The proportionality would be retained only if $\tau(v)$ were independent of $v$ instead of sharply peaked at line center. Since the spectral shape of $I_{\text {th }}$ follows the same Doppler profile as $\kappa_{0}$, straightforward algebra yields

$$
\mathscr{R}(v)=\frac{\Delta v_{B} \cos \theta}{1+\tau_{0} \exp \left(-x^{2}\right)} .
$$

The $\mathscr{R}$ profile is constant only at $\tau_{0}=0$, i.e., only for the input radiation. The amplification process destroys the profile flatness even though it only amplifies the intensity without affecting the polarization. When $\tau_{0}>1, \mathscr{R}$ assumes instead a Gaussian absorption shape. This happens because the amplification is centered on $v_{0}$; therefore the $I^{ \pm}$components are amplified more strongly on their inner shoulders, the ones closer to $v_{0}$. Whereas this preserves the general symmetry principle of equation (13), as it must, the invariance under frequency shifts of equation (10) is destroyed because $I^{ \pm}(v)$ are no longer symmetric about their centroids at $v_{ \pm}=v_{0} \pm \Delta v_{B}$.

\subsection{Saturated Masers}

When the maser is saturated $\left[J(v)>J_{s}\right]$, the radiative transfer equation for the intensity of the dominant ray of the polarization solution becomes

$$
\frac{d I(v)}{d \ell}=3 \pi J_{s} \frac{\kappa_{0}(v)}{\Omega(v)}
$$

where $\Omega(v)$ is the maser beaming angle, which in general may vary with frequency shift from line center (Elitzur 1990). Therefore, the profile of $I(v)$ has the spectral shape of the ratio $\kappa_{0}(v) / \Omega(v)$.

The frequency variation of $\Omega(v)$ depends on the type of maser amplification, which is controlled by the geometry (Elitzur, Hollenbach, \& McKee 1992). In matter-bounded masers, whose prototypes are filaments, the beaming angle is independent of frequency and $I(v) \propto \kappa_{0}(v)$. In amplification-bounded masers the beaming angle varies with frequency shift from line center according to dimensionality. In the prototype planar maser, a saturated disk, $\Omega(v) \propto$ $1 / \kappa_{0}(v)$ and $I(v) \propto\left[\kappa_{0}(v)\right]^{2}$. And in the prototype threedimensional maser, a saturated sphere, $\Omega(v) \propto 1 /\left[\kappa_{0}(v)\right]^{2}$, so $I(v) \propto\left[\kappa_{0}(v)\right]^{3}$. In summary, the intensity spectral profile of a saturated maser obeys

$$
I(v) \propto\left[\kappa_{0}(v)\right]^{p},
$$

where $p$ is the dimensionality of the geometry: 1 for filaments, 2 for planar masers, and 3 for spherelike configurations. Therefore $I^{\prime} / I=p \kappa_{0}^{\prime} / \kappa_{0}$ and the $\mathscr{R}$ profile of a saturated maser is

$$
\mathscr{R}(v)=\frac{8 \Delta v_{B}}{3 p \cos \theta} .
$$

The unsaturated profile $\Psi$ is replaced by the constant $1 / p$ during the saturation process, which reflects the rebroadening of the maser line. Similar to thermal radiation, $\mathscr{R}$ is constant across the saturated maser line, only the value of this constant is different. For a given magnetic field and propagation at the smallest angle allowed for maser polarization, $\theta=\cos ^{-1}(2 / 3)^{1 / 2}=35^{\circ}$, the proportionality constant for filamentary masers is 4 times larger than for thermal radiation (twice as large for disks, 1.3 times for spheres). In other words, masers require smaller fields to produce the same circular polarization as thermal radiation. The disparity between the two cases increases with $\theta$ in proportion to $1 / \cos ^{2} \theta$.

The saturation effect was introduced here in its standard form. Strictly, this form applies only to linear masers; threedimensional effects lead to a more complex form (Litvak 1973; Bettwieser \& Kegel 1974; Neufeld 1992). However, because of the tight beaming of maser radiation, the standard form provides an adequate approximation around line center, failing only in the extreme wings $(x>2$; Elitzur 1994) of saturated masers, and its use is justified in the present analysis. In addition, frequency redistribution has been neglected. Incorporation of this important ingredient into maser theory is sufficiently difficult that it has not yet been fully accomplished even for scalar masers. In keeping with common practice, it has been neglected in this first study. Also, the maser polarization problem was solved only for a $J=1 \rightarrow 0$ transition. However, when $x_{B} \ll 1$ the 
solution holds for all spins (E96) and thus is applicable here. Note, though, that this result holds only for isotropic pumping. Pumping schemes that will introduce $m$ dependence would require separate handling.

\section{RESOLVED ZEEMAN PATTERN: $x_{B}>1$}

When $x_{B}>1$ the Zeeman components separate and the radiation displays three fully polarized lines whose linear and circular fractional polarizations are constant across each component. The circular polarizations of the two $\sigma$ components, centered on $v_{ \pm}=v_{0} \pm \Delta v_{B}$, are

$$
v^{ \pm}= \pm \frac{2 \cos \theta}{1+\cos ^{2} \theta}
$$

whatever the degree of saturation (Goldreich, Keeley, \& Kwan 1973; E96; see Field \& Gray 1994 for the approach to this solution of amplified unpolarized background radiation). In fact, this result follows from general symmetry properties and holds also for thermal radiation, as can be easily verified from expressions listed in Crutcher et al. (1993). Although the polarization is constant across each individual profile, it maintains the reflection symmetry of equation (13) since $v^{-}=-v^{+}$. Independent of the nature of the radiation, $V(v)$ is proportional to $I(v)$ across each component, not to $I^{\prime}(v)$. The ratio profile now obeys $\mathscr{R}=v^{ \pm} I / I^{\prime}$, which leads to

$$
\mathscr{R}(v)=-\frac{v^{ \pm}\left(\Delta v_{\mathrm{D}}\right)^{2}}{2\left(v-v_{ \pm}\right)} \times \begin{cases}1 & \text { thermal } \\ \Psi(v) & \text { unsaturated maser } \\ 1 / p & \text { saturated maser }\end{cases}
$$

if $\kappa_{0}$ and the thermal radiation follow a Doppler profile with width $\Delta v_{\mathrm{D}}$. In the profile $\Psi$ of each unsaturated component, the intensity is normalized to its line center magnitude for that component (cf. eq. [19]).

The ratio profiles for $x_{B} \ll 1$ and $x_{B}>1$ are fundamentally different from each other. In one case $\mathscr{R}$ is symmetric in reflections about the center of the single observed line, in the other it is antisymmetric about the center of each observed $\sigma$ component at $v_{ \pm}$. This difference provides a decisive observational test to determine whether the Zeeman splitting is larger or smaller than the line width.

\section{OBSERVATIONAL IMPLICATIONS}

Spectral analysis of circular polarization provides a new powerful tool for maser studies. Comparison of the profiles of $V(v)$ and $I^{\prime}(v)$ offers sensitivity to details at the level of $\sim x_{B}$ and has long been utilized in studies of thermal radiation. The maser analysis can be readily performed with the techniques developed for the thermal case (see, e.g., Troland $\&$ Heiles 1982) where the observed $V$ spectrum is fitted as

$$
V(v)=a I(v)+b I^{\prime}(v)
$$

and $a$ and $b$ are free parameters. The $a I$ term is introduced to account for differences in instrumental sensitivity in left and right circular polarization. The parameter $a$ has no bearing on the source properties, it is merely adjusted to make $V-a I$ antisymmetric about line center. The next step is to fit $V-a I$ to the intensity derivative $I^{\prime}$, which produces the meaningful parameter $b=\Delta v_{B} \cos \theta$ (eq. [12]).

In the case of maser radiation, various mechanisms can filter one sense of circular polarization in the source itself (see, e.g., § 6.7 of Elitzur 1992, and references therein). Then the observed $V$ profile may not be perfectly antisymmetric even before it reaches the detection instruments. Similar to instrumental effects, the left-right asymmetry introduced by such filters can be handled by fitting the observed $V$ profile of an unsaturated maser as

$$
V(v)=a I(v)+\frac{b I^{\prime}(v)}{c+\ln \left[I(v) / I\left(v_{0}\right)\right]}
$$

(eq. [18]), with $a$ adjusted to make $V-a I$ antisymmetric about line center. Fitting the resulting antisymmetric profile produces the adjustable parameters $b=8 \Delta v_{B} / 3 \cos \theta$ and $c=\tau_{0}$, the maser optical depth at line center. This is the first method to directly determine the maser optical depth for self-amplification and the intrinsic velocity Doppler width (obtained from the spectral shape of $\ln I$ ).

When the maser is saturated $\mathscr{R}$ is flat, so the $V$ profile is fitted as in the thermal case only $b=8 v_{B} / 3 p \cos \theta$ (eq. [26]). The parameter $p$ is determined by the maser geometry (it is 1 for filaments, 2 for planar masers, and 3 for threedimensional masers), and this cannot be found directly from observations. However, strong masers are unlikely to be spherical, so $p=3$ can usually be dismissed. In particular, the relevant geometry for shock generated masers, applicable to water masers as well as the $1720 \mathrm{MHz}$ masers at the Galactic center (Yusef-Zadeh, Uchida, \& Roberts 1995; Frail et al. 1996; Yusef-Zadeh et al. 1996), is likely to be planar or filamentary and $p$ is either 2 or 1 . Another handle on $p$ can come from the line width $\Delta v_{m}$ of the observed maser intensity. When $\kappa_{0}$ has a Doppler shape with width $\Delta v_{\mathrm{D}}$, equation (25) shows that

$$
\Delta v_{m}=\frac{\Delta v_{\mathrm{D}}}{\sqrt{p}}
$$

and the line width observed in disk masers is only $70 \%$ of the Doppler width. A line narrower than the expected thermal width in a saturated maser can be taken as indication of planar rather than filamentary geometry.

The distinct shape of the unsaturated $\mathscr{R}$ profile, evident in Figure 1, provides the first direct method to determine the saturation stage of a maser. Detection of the predicted logarithmic variation across the line would provide a unique, unambiguous signature of unsaturated maser operation. However, a flat $\mathscr{R}$ cannot immediately be taken as conclusive evidence for saturated operation; within the observational errors, such behavior can always be attributed to sufficiently large $\tau_{0}$. If $\mathscr{D}$ denotes the dynamic range of intensity measured across an unsaturated maser line, the expected relative variation of $\mathscr{R}$ is $\left(1 / \tau_{0}\right) \ln \mathscr{D}$. The observed profile could be indistinguishable from a constant if this variation is smaller than the observational error $\epsilon$, namely, if

$$
\tau_{0}>\frac{1}{\epsilon} \ln \mathscr{D} .
$$

A flat $\mathscr{R}$ only implies a lower limit on $\tau_{0}$. However, if the maser is indeed unsaturated, then there is also an upper limit on $\tau_{0}$. In this case, the measured brightness temperature at line center is $T_{b}=T_{x} \exp \tau_{0}$, where $T_{x}$ is the maser excitation temperature. This quantity in turn is related to the fractional inversion $\eta=\left(n_{2}-n_{1}\right) /\left(n_{2}+n_{1}\right)$ via $T_{x}=h v_{0} / 2 k \eta$ (e.g., Elitzur $1992 \S 4.2$ ). Therefore $\tau_{0}=\ln$ $\left(2 \eta k T_{b} / h v_{0}\right)$, and an absolute upper limit on $\tau_{0}$ is obtained by inserting in this expression $\eta=1$, the maximum theoretical 
value, yielding

$$
\tau_{0}<\ln \frac{2 k T_{b}}{h v_{0}} .
$$

The optical depth of an unsaturated maser is always bounded by the observed brightness temperature. The maser can display a flat $\mathscr{R}$ profile only if this upper limit is compatible with the previous lower limit.

To summarize the observation analysis procedure: The free parameter $a$ is varied until the combination $V-a I$ of the observed $V$ and $I$ profiles is antisymmetric about line center, then the ratio $I^{\prime} /(V-a I)$ is formed. If this ratio displays linear variation with $\ln I$ across the line then the maser is unsaturated and the slope and intercept of the linear fit determine $B / \cos \theta$ and $\tau_{0}$. If, instead, the ratio $I^{\prime} /(V-a I)$ is constant across the line then the situation depends on the last two bounds. If these bounds are incompatible, the maser is saturated. That is, if the fitting error $\epsilon$ and the observed brightness temperature $T_{b}$ and dynamic range $\mathscr{D}$ obey

$$
\ln \mathscr{D}>\epsilon \ln \frac{2 k T_{b}}{h v_{0}},
$$

yet $I^{\prime} /(V-a I)$ is constant across the line, then the maser must be saturated. When the reverse relation exists between $\mathscr{D}, \epsilon$, and $T_{b}$, the saturation stage is unsettled; the data lack the sensitivity to distinguish between the $\mathscr{R}$ profiles of saturated and unsaturated maser operation.

Observational data that obey the last inequality are of sufficient quality to determine conclusively whether the maser is saturated or not. The recent VLA polarization data of OH $1720 \mathrm{MHz}$ masers near the Galactic center by YusefZadeh et al. (1996) are the first to meet this criterion. The brightness temperatures of all resolved Galactic $\mathrm{OH}$ masers $\left(h v_{0} / k=0.08 \mathrm{~K}\right)$ always obey $T_{b} \gtrsim 10^{10} \mathrm{~K}$. If any such maser is unsaturated, the upper bound of equation (33) implies that $\tau_{0}<26$ (a realistic estimate for the inversion, $\eta \lesssim 10 \%$, would reduce this bound to $\tau_{0}<24$ ). The $\mathscr{R}$ profiles of the Galactic center masers were fitted as constants by Yusef-Zadeh et al. (1996) and the errors listed for the magnetic fields are less than $10 \%$ in all sources, as low as $3 \%$ in the strongest (source A). These are essentially error estimates of the quality of the fits to flat ratio profiles, thus $\epsilon \sim 0.03-0.1$. The resulting fits match the observations all the way to the noise level of $\sim 10 \mathrm{mJy}$ for an intensity dynamic range $\mathscr{D} \gtrsim 1000$ in the strongest source and no less than $\sim 20$ in the weakest (source F). Therefore, from equation (32) the lower limits on $\tau_{0}$ range from 30 to 230 and are incompatible with the upper limit for unsaturated $\mathrm{OH}$ masers. This conclusion can be strengthened by a proper analysis that would test the hypothesis of an unsaturated $\mathscr{R}$ spectral shape with the observations. Such analysis should produce constraints that are even tighter and more secure. Still, it seems safe to conclude already on the basis of the current analysis that at least the strongest masers, and quite possibly all of them, are saturated. Previously, the strongest evidence for saturation was provided by $\mathrm{OH} 1612$ MHz masers in late-type stars (Elitzur 1992, § 8.6). This maser emission follows the temporal variation of the IR radiation, the presumed pump, and the similar amplitudes indicate a linear relation between pump and maser intensity, as expected in saturated operation. Although this evidence seems persuasive, it is indirect as it requires an assumption, however plausible, regarding the pump mechanism. In contrast, the new evidence is direct, obtained from the relation between $I$ and $V$, two maser intensities measured independently.

Detailed profile analysis of circular polarization also enables direct, unambiguous determination of the relation between the magnitudes of the Zeeman splitting and the line Doppler width. Substantial circular polarization $(50 \%$ and higher) has long been observed in $\mathrm{OH}$ maser emission from late-type stars (e.g., Reid et al. 1979; Claussen \& Fix 1982; Cohen et al. 1987). In the absence of theory for $x_{B}<1$ at the time, this was taken as a signature of $x_{B} \gtrsim 1$, implying magnetic fields of at least $\sim 1-10 \mathrm{mG}$. However, this polarization often appears as sharp reversals between adjacent narrow spectral components of the parameter $V$, as expected for $x_{B}<1$. Analysis in terms of the theory for $x_{B}<1$ yields instead fields of only $\sim 0.1 \mathrm{mG}$ (E96), so it is important to determine conclusively what is the relevant domain of $x_{B}$. The new profile analysis provides a simple, reliable method to resolve this issue. If the $V$ profile can be fitted across each component as $V=a I$ without residuals then $x_{B}>1$. If, on the other hand, the residuals are significant and $V-a I$ is antisymmetric around line center then $x_{B}<1$.

Finally, circular polarization analysis, of either thermal or maser radiation, can never fully determine the magnetic field because the propagation angle $\theta$ is not known. However, in the case of maser radiation $\theta$ can be determined from linear polarization measurements; the polarization solution shows that both circular and linear polarizations are generated when $x_{B} \ll 1$, and to leading order in $x_{B}$ the linear polarization is a unique function of $\theta$ (E96, eq. [4.14]). Indeed, strong linear polarization has been recently reported for the $1720 \mathrm{MHz}$ masers by Claussen et al. (1997). Although Faraday rotation can reduce the linear polarization, even eliminate it on occasion, study of linear polarization is an important task for observations.

Discussions with Tom Troland and Farhad Yusef-Zadeh, and the partial support of NASA grants NAG 5-3010 and NAG 5-7031 are gratefully acknowledged.
Bettwieser, E. V., \& Kegel, W. H. 1974, A\&A, 37, 291

Claussen, M. J., \& Fix, J. D. 1982, ApJ, 263, 153

Claussen, M. J., et al. 1997, ApJ, 489, 143

Cohen, R. J., et al. 1987, MNRAS, 225, 491

Crutcher, R. M., et al. 1993, ApJ, 407, 175

Elitzur, M. 1990, ApJ, 363, 638

1992, Astronomical Masers (Dordrecht: Kluwer) 1994, ApJ, 422, 751 1996, ApJ, 457, 415 (E96)

Elitzur, M., Hollenbach, D. J., \& McKee, C. F. 1992, ApJ, 394, 221

Fiebig, D., \& Gusten, R. 1989, A\&A, 214, 333

Field, D., \& Gray, M. D. 1994, A\&A, 292, 271

\section{REFERENCES}

Frail, D. A., Goss, W. M., Reynoso, E. M., Giacani, E. B., Green, A. J., \& Otrupeck, R. 1996, AJ, 111, 1651

Goldreich, P., Keeley, D. A., \& Kwan, J. Y. 1973, ApJ, 179, 111

Litvak, M. M. 1973, ApJ, 182, 711

. 1975, ApJ, 202, 58

Neufeld, D. 1992, ApJ, 393, L37

Reid, M. J., et al. 1979, ApJ, 227, L89

Troland, T., \& Heiles, C. 1982, ApJ, 252, 179

Yusef-Zadeh, F., Roberts, D. A., Goss, W. M., Frail, D. A., \& Green, A. J. 1996, ApJ, 466, L25

Yusef-Zadeh, F., Uchida, K. I., \& Roberts, D. A. 1995, Science, 270, 1801

Zell, P. J., \& Fix, J. D. 1991, ApJ, 369, 506 